\title{
Creencias, actitudes y valores relacionados a la infancia y trabajo infantil en madres de Lima Metropolitana ${ }^{1}$
}

\author{
Ana Simón ${ }^{2}$, Henry Guillén ${ }^{3}$ y Rosa María Cueto ${ }^{4}$ \\ Pontificia Universidad Católica del Perú
}

\begin{abstract}
La presente investigación buscó identificar y analizar las creencias y actitudes entorno a la infancia y el trabajo infantil y su relación con los valores individuales en 12 madres pertenecientes a los distritos de Villa El Salvador y San Juan de Lurigancho en Lima Metropolitana. Para ello, se construyó una entrevista semi-estructurada. Se obtuvieron como resultados que las entrevistadas vinculan la infancia con características y actividades relacionadas principalmente con la recreación y la educación, evalúan al trabajo infantil de forma negativa y ambivalente, influenciadas por el contexto inseguro en el que viven. Además, evidencian como base de sus creencias y actitudes sobre la infancia y trabajo infantil, valores como trabajo, progreso, responsabilidad y filiación.

Palabras clave: creencias, actitudes, valores, infancia, trabajo infantil
\end{abstract}

Beliefs, Attitudes and values of mothers related to Childhood and Child Labor in Lima The present research sought to identify and analyze the beliefs and attitudes towards childhood and child labor and the association with individual values of mothers from the districts of Villa El Salvador and San Juan de Lurigancho in Lima Metropolitana. Twelve mothers were interviewed using a semi-structured interview. Results indicate that participants link childhood with characteristics and activities related primarily to recreation and education. Likewise, they evaluate child labor in a negative and ambivalent way, which is influenced by living in an unsafe context. Their beliefs and attitudes towards childhood and child labor are rooted in values such as work, progress, responsibility and filiation.

Keywords: Beliefs, attitudes, values, childhood, child labor

1 Investigación financiada por la Pontificia Universidad Católica del Perú a través de la Dirección de Gestión de la Investigación (DGI).

2 Licenciada en Psicología Social por la Pontificia Universidad Católica del Perú, pre-docente de la misma casa de estudios. Dirección postal: Av. Universitaria 181, San Miguel, Lima 32, Perú. Contacto: ana.simon@pucp.pe. https://orcid.org/0000-0003-2947-6435

3 Magister en Desarrollo Humano por la Pontificia Universidad Católica del Perú, asistente de docencia y miembro del Grupo de Psicología Política y Social de la misma casa de estudios. Dirección postal: Av. Universitaria 181, San Miguel, Lima 32, Perú. Contacto: hguillen@ pucp.pe. https://orcid.org/0000-0001-6574-8077

4 Doctora en Psicología por la Pontificia Universidad Católica del Perú, docente y miembro del Grupo de Psicología Política y Social de la misma casa de estudios. Dirección postal: Av. Universitaria 181, San Miguel, Lima 32, Perú. Contacto: rcueto@pucp.pe. https://orcid. org/0000-0003-3549-2001 


\section{Crenças, Atitudes e valores relacionados à Infância e Trabalho Infantil em Lima}

A pesquisa atual buscou identificar e analisar crenças e atitudes em relação à infância e trabalho infantil e sua relação com valores individuais em 12 mães pertencentes aos distritos de Villa El Salvador e San Juan de Lurigancho, na metrópole de Lima. Para isso, foi construída uma entrevista semiestruturada. Foram obtidos resultados onde as entrevistadas vinculam a infância com características e atividades relacionadas principalmente à recreação e educação; Elas avaliam o trabalho infantil de forma negativa e ambivalente, influenciadas pelo contexto inseguro em que vivem. Elas também mostram valores como Trabalho, Progresso, Responsabilidade e Filiação como base para suas crenças e atitudes sobre infância e trabalho infantil.

Palavras-chave: Crenças, atitudes, valores, infância, trabalho infantil

\section{Croyances, attitudes et valeurs liées a l'enfance et le travail des enfants à Lima Métropolitaine}

Cette recherche visait à identifier et analyser les croyances et les attitudes concernant l'enfance et le travail des enfants et sa relation avec les valeurs individuelles de 12 mères appartenant aux districts de Villa El Salvador et San Juan de Lurigancho dans la région métropolitaine de Lima. Pour cela, une interview semi-structurée a été construite. Les résultats obtenus ont montré que les interviewées établissent un lien entre l'enfance et les caractéristiques et activités liées principalement aux loisirs et à l'éducation; elles évaluent négativement et ambivalemment le travail des enfants, influencées par le contexte d'insécurité dans lequel elles habitent. En outre, elles montrent à la base de leurs croyances et attitudes concernant l'enfance et le travail des enfants, des valeurs telles que le travail, le progrès, la responsabilité et la filiation.

Mots-clés: croyances, attitudes, valeurs, enfance, travail des enfants 


\section{Trabajo infantil e infancia}

Estimaciones a nivel mundial refieren que 168 millones de niños, nińas y jóvenes de entre 5 y 17 años realizan trabajo infantil; es decir, un $11 \%$ de la población total de ese rango de edad. De ellos, 85 millones realizan las peores formas del trabajo infantil, es decir un 50.6\% (Organización Internacional del Trabajo [OIT], 2013). En América Latina y El Caribe, 12,5 millones de niños, niñas y jóvenes trabajan, que es un $8.8 \%$ de dicha población (OIT, 2013). Y en el Perú, el 23.4\% de niños, niñas y jóvenes de entre 6 y 17 ańos trabajan, es decir, 1.65 millones, de los cuales, el $41.3 \%$ residen en zonas urbanas y el $7 \%$ reside en Lima (Ministerio de Trabajo y Promoción del Empleo [MTPE], 2012).

Sobre el trabajo infantil, podría decirse que definirlo genera posiciones divididas entre quienes lo investigan, las cuales se vinculan principalmente a su conceptualización, al rango de edad mínimo consentido, al tiempo mínimo considerado aceptable de horas al día dedicadas al trabajo, a la inclusión o no de los quehaceres domésticos dentro la definición de trabajo infantil, entre otros aspectos (Liebel, 2003). Sin embargo, las coincidencias entre investigadores permiten definir al trabajo infantil como la participación de niños, niñas y adolescentes en actividades que contribuyan con la economía familiar, sin tomar en consideración el estado ocupacional, es decir, indiferentemente de si es o no trabajo asalariado, independiente o dependiente, trabajo familiar no remunerado, etc. (OIT, 2007; Silva, 2010; Silva, 2013).

En este sentido, un concepto directamente vinculado al trabajo infantil es el de infancia, el cual puede ser entendido como un periodo medido por un intervalo de edad que es socialmente consensuado (Alzate, 2003), y en el Perú dicho intervalo abarca desde que la persona nace hasta los doce años (Sandoval, 2011). La infancia, es considerada una etapa de suma importancia en el desarrollo pues marca la vida a futuro de una persona al ser un periodo donde se forman soportes 
intelectuales y afectivos (Arrizaga, 2010). Sobre esta consideración, en relación con el trabajo infantil, se plantean dos posturas. Una de ellas atribuye a la infancia como un momento especial en el desarrollo del sujeto, viendo a los niños y nińas como seres vulnerables y enfatizando la necesidad de tener cuidados especiales con ellos, por lo que se consideraría el trabajo infantil como "enemigo de los niños" (Rausky, 2008). Mientras que otra postura, entiende la infancia desde una perspectiva desde la cual los niños y las niñas son seres con igualdad de derechos, con capacidad para opinar y decidir sobre circunstancias que afectan su vida y su futuro, lo cual significaría que tienen la posibilidad de decidir cuándo, cómo y en qué trabajar (Liebel, 2003).

\section{Creencias, actitudes y valores}

Las creencias son un resumen de la visión que tienen las personas sobre la organización social, la cultura y la forma en que viven sus relaciones interpersonales; las cuales pueden cambiar en función del contexto espacial y temporal del sujeto (Garzón, 2012). Respecto a las creencias parentales, estas son definidas por Lightfoot y Valsiner (1992) como "productos psicológicos del proceso de comunicación cultural” (p. 408), las que funcionan como un organizador mental de las acciones que los padres y madres pueden llegar a mostrar en relación a sus formas de crianza (Oliveira \& Uchoa, 2010).

Por otro lado, las actitudes conforman un concepto que se vincula directamente a las creencias; representando una evaluación integrada de las cogniciones (creencias) y los afectos experimentados en relación a un objeto (Holland, Meertens \& Van Vugt, 2002). Dicho objeto actitudinal puede ser cualquier elemento que la persona tenga en mente, desde lo concreto hasta lo abstracto, lo cual incluye cosas, personas, grupos, ideas, etc. (Bohner \& Dickel, 2011).

Por su parte, los valores se definen como construcciones cognitivas (creencias) conectadas a emociones que funcionan como guías de conducta que trascienden situaciones específicas, como vías de expresión cognitiva de necesidades (Gouveia, Santos, Milfont, Fischer, Clemente \& Espinosa, 2010) y como criterios de evaluación de acontecimientos, 
conductas y personas. Dichos valores se presentan en diferentes grados de importancia para la persona, en relación a su cultura y creencias (Schwartz, 2007).

Sobre lo mencionado, Gouveia (2003) plantea un modelo de valores que integra anteriores modelos teóricos y conceptualiza a los valores en base a dos funciones consensuales. La primera, vinculada a la orientación del valor en su función como guía de conductas tomando tres posibilidades: social, central y personal; y la segunda, vinculada a la motivación subyacente a cada valor en su función de expresar necesidades humanas, de la cual se derivan valores materialistas (pragmáticos) o humanitarios (idealistas) (Gouveia, Fischer \& Milfont, 2009).

Al unir estas dos funciones principales, se obtienen seis subfunciones, las cuales son: Experimentación, que constituye la necesidad fisiológica de satisfacción y hedonismo, representando valores como sexualidad, placer y emoción; realización; que representa las necesidades de autoestima, relacionado con valores de prestigio, éxito y poder; existencia, que hace referencia a las necesidades fisiológicas y las necesidades de seguridad, las cuales se relacionan con valores tales como salud, supervivencia y estabilidad emocional; suprapersonal, la cual refiere necesidades de cognición y estéticas, asociado a valores como conocimiento, madurez y belleza; interactiva, la cual figura la necesidad de pertenencia, afiliación y amor, vinculándose a valores como afectividad, convivencia y apoyo social; y normativa, que constituye la necesidad de control, respetando normal sociales, vinculados a valores de tradición, obediencia y religiosidad (Gouveia, et al., 2010).

Sobre la relación entre actitudes, creencias y valores, algunos estudios han encontrado que estos constructos se vinculan en la medida que son adquiridos en la socialización primaria, siendo usualmente transmitidos de cuidadores(as) a hijos(as) (Ward, Bochner \& Furnham, 2001). Sin embargo, los valores tienden a ser más estables que las actitudes, pues son más resistentes al cambio (Ward et al., 2001), siendo orientadores de las actitudes, ya que estas últimas pueden ser influenciadas por aspectos situacionales (Kilby, 1993). 


\section{Creencias y actitudes en torno a la infancia y al trabajo infantil}

En el Perú, un estudio hecho por la OIT (2007) que buscó conocer las actitudes, percepciones y conocimientos de los peruanos sobre el trabajo infantil, evidenció una baja o nula tolerancia hacia el trabajo infantil y una percepción negativa sobre sus efectos, ya que se cree perjudica el rendimiento y la permanencia del niño en el colegio (Rausky, 2008). Sin embargo, se evidenció también una visión del trabajo infantil como fenómeno inevitable, principalmente a causa de la pobreza, y se vinculó al Estado como principal actor llamado a llevar a cabo acciones de intervención directa (OIT, 2007).

Enfocándonos en los padres, madres o responsables de la nińez trabajadora, algunas investigaciones afirman que una de las justificaciones vinculadas al trabajo del menor es la falta de ingresos económicos en la familia; siendo así que los beneficios cualitativos y cuantitativos del trabajo infantil, como la adquisición de habilidades, actitudes y disciplina, y el ingreso económico respectivamente, serían más valorados que la educación de los hijos, pues esta sería vista como inaccesible, de poca calidad o ineficaz (del Río \& Cumsille, 2008; OIT, 2003). Otras justificaciones del trabajo infantil están relacionadas al desarrollo de valores en el menor como la autonomía, la responsabilidad, la disciplina, la tenacidad y la capacidad de afrontar dificultades y problemas (Padrón \& Román, 2013). Esto también implicaría la adquisición de experiencia, madurez y la asunción de un rol significativo en la estructura familiar y comunitaria (Calderón, 2004; Padrón \& Román, 2013). Además, el trabajo sería percibido como un factor protector ante situaciones y el desarrollo de conductas de riesgo latentes en el contexto (Padrón \& Román, 2013).

En contextos vulnerables, la forma en cómo las personas entiendan distintas problemáticas podría variar de acuerdo a su estructura de creencias, actitudes y valores subyacentes a ellas. La vulnerabilidad puede ser vista como una situación riesgosa que suele ser definida por circunstancias relacionadas a las condiciones sociales, donde usualmente subyacen la desigualdad, la poca confianza entre sus habitantes, autoridades poco preocupadas y un poco acceso a diferentes recursos 
(Ruiz, 2012). Con ello, el trabajo infantil, responde en parte, a este contexto de vulnerabilidad en el que el menor y su familia se encuentran inmersos (OIT, 2013). En esta línea, se ha reportado que los padres de familia de nivel socioeconómico bajo ven al trabajo infantil solo como aquellas actividades que refieran un ingreso monetario para la familia; las actividades realizadas dentro y para la familia son vistas como formativas y de apoyo al hogar, no limitantes del desarrollo del menor (UNICEF, 2004 citado por Román \& Murillo, 2013; Silva, 2010).

De lo expuesto, cabe resaltar la falta de información en el Perú sobre el cuidador principal de infantes en contextos vulnerables y su manera de entender y evaluar la infancia y el trabajo infantil. Siendo de suma importancia conocer y dar a conocer sus creencias, actitudes y valores subyacentes, en torno a esta problemática, ya que, como parte de su sistema psicológico, estos componentes se vinculan con su accionar y con la transmisión de estos modos de vivir a sus hijos e hijas, los cuales afectan significativamente su desarrollo (Oliveira \& Uchoa, 2010). En este sentido, las creencias y actitudes que tienen los cuidadores sobre la infancia y sobre el trabajo infantil, así como los valores que poseen, podrían condicionar las expectativas que llegarían a tener sobre los niños y niñas (Romero, Amar, Palacio, Madariaga, Sierra \& Quintero, 2012). Así también, funcionarían como mecanismos de decisión respecto al incentivo que se da al menor para colaborar con la familia realizando actividades que contribuyan al hogar, ya sea generando ingresos o apoyando en casa (del Rio \& Cumsille, 2008). En por ello, que el objetivo general del presente estudio es analizar las creencias y actitudes sobre la infancia y el trabajo infantil y su relación con los valores individuales en madres que viven un contexto de vulnerabilidad en Lima Metropolitana.

\section{Método}

\section{Participantes}

Las participantes fueron 8 madres de familia residentes del sector 8 del distrito de Villa El Salvador (VES) y 4 madres residentes de 
Jicamarca del distrito de San Juan de Lurigancho (SJL) en Lima Metropolitana. Estos distritos presentan altos índices de pobreza (INEI, 2015). Además de indicadores poco favorables en confianza hacia las instituciones, confianza interpersonal, percepciones de seguridad, entre otros (Encuesta Lima Cómo Vamos, 2015). Estas características coinciden con la descripción que se hace sobre contextos de vulnerabilidad (Alegre \& Bielich, 2015). Las edades de las entrevistadas oscilan entre los 29 y los 55 años. Sobre el nivel educativo, 2 tienen primaria incompleta, 1 primaria completa, 2 secundaria incompleta, 6 secundaria completa y 2 educación técnica incompleta. Del total de entrevistadas, 10 nacieron fuera de Lima y 2 en Lima. Las participantes residen en su comunidad, como mínimo, desde hace 10 años. Dentro de las actividades que realizan principalmente se encuentran las labores domésticas, trabajo en casa para terceros y trabajos independientes. Respecto al número de hijos e hijas, las entrevistadas tienen un promedio de 2.58 hijos(as) con edades entre los 5 y 16 años.

La selección de los participantes fue de tipo intencional (Hernández, Fernández \& Baptista, 2014), en este sentido se contactó a las participantes en función de su disponibilidad y voluntad para participar de este estudio. En este caso, fueron solo madres quienes contaban con tiempo y disponibilidad dado, ya que los hombres se encontraban trabajando en los momentos que se dispuso realizar el trabajo de campo.

\section{Técnicas de recolección de información}

El presente estudio tuvo una aproximación cualitativa. Para la recolección de la información se elaboró una guía de entrevista semiestructurada basada en la investigación teórica, en este sentido, la guía de entrevista fue construida tomando en cuenta preguntas que facilitaron el poder repreguntar ante información que necesitó ser profundizada en los siguientes temas:

- Valores individuales: se consultó a las participantes con respecto a elementos que se consideren valorables en una persona en sus contextos. 
- Infancia: se buscó conocer el significado que la entrevistada le da a la infancia, así como las características que le atribuye a un niño(a), haciendo énfasis en las actividades realizadas por ellos y los roles que desempeñan.

- Trabajo infantil: se exploró acerca del significado, características, actividades, razones y consecuencias del trabajo infantil.

\section{Procedimiento}

En cuanto al desarrollo de la entrevista, se procedió primero a contactar a los padres/madres dentro de las instituciones educativas de sus hijos(as), donde se les explicó la naturaleza de la investigación, así como los objetivos de la misma; después se procedió a anotar los datos de las personas dispuestas a participar. Posteriormente, el entrevistador contactó a cada uno de los interesados, confirmando su participación y conviniendo la fecha, lugar y hora de la entrevista.

Para iniciar la entrevista, se coordinó que esta se desarrollara en la casa de las participantes. Asimismo, las entrevistadas firmaron un consentimiento informado donde se les explicaba las consideraciones éticas, así como los objetivos de la investigación. Por último, se desarrolló la entrevista, de acuerdo a los temas determinados en la guía. Cada entrevista tuvo una duración de aproximadamente 45 minutos. Cabe mencionar que las entrevistas fueron grabadas con el consentimiento de la participante.

\section{Análisis de la información}

Posteriormente se transcribieron las entrevistas de forma completa. Luego se realizó el análisis de contenido (Graneheim \& Lundman, 2004), utilizando como base el programa Atlas.ti 7.5.4, a partir del cual se formaron categorizaciones a partir de las variables y ejes temáticos de la guía de entrevista en relación a la infancia y trabajo infantil. A partir de las cuales se generaron subcategorías y finalmente, unidades de significado. Por otro lado, para determinar en qué subfunción se ubicaban los valores expresados por las participantes, se analizaron 
los mismos según su tipo de orientación y motivación, de acuerdo al modelo de Gouveia et al. (2010). Finalmente, los valores identificados y las unidades de significado fueron relacionadas entre en un esquema elaborado a partir de las respuestas. Cabe resaltar que, para garantizar un apropiado proceso metodológico dentro de la investigación, la codificación de la información fue triangulada por los investigadores para contrastar, confirmar y validar los datos obtenidos (Thurmond, 2001).

\section{Resultados y discusión}

A continuación, se presentarán los resultados y la discusión. En primer lugar, se expondrán las categorías emergentes sobre el concepto de infancia, las características de infantes trabajadores y no trabajadores, las actividades relacionadas a la infancia y las expectativas que se tienen sobre la misma. Luego se presentarán las categorías emergentes sobre las actividades relacionadas y no relacionadas al trabajo infantil, las causas, las consecuencias, y las actitudes sobre el trabajo infantil. Posteriormente, se mostrará cómo se relaciona el contexto de vulnerabilidad con algunas de las actitudes y creencias sobre la infancia y trabajo infantil. Finalmente, se expondrá la estructura de valores de las madres entrevistadas y cómo estos estarían a la base de sus creencias y actitudes sobre la infancia y el trabajo infantil.

\section{Infancia y trabajo infantil en un contexto de vulnerabilidad}

La infancia puede entenderse como una etapa en la vida que es medida por un intervalo de edad consensuado socialmente (Alzate, 2003). En este sentido, la infancia es definida como un periodo en la vida de una persona que toma lugar desde que nace hasta los doce años (Sandoval, 2011), esto coincide con lo reportado por las madres entrevistadas, quienes, en general, atribuyen este mismo intervalo a la infancia.

Siguiendo esta línea, cabe mencionar, que cada cultura y sociedad define de manera implícita o explícita el significado que tiene la 
infancia y cuáles son las características atribuidas a este periodo de vida (Casas, 1998). En este caso, las madres entrevistadas le atribuyen tanto a los(as) niños(as) en general como a los(as) niños(as) no trabajadores en específico, características tales como: ser juguetones, alegres, tímidos, educados y dependientes. Esto se aprecia en la siguiente viñeta: "Un niño no trabajador es feliz, porque estaría viviendo su niñez, jugando, divirtiéndose, iría al colegio" (madre, 36 años, VES). En oposición a esto, las características asociadas a un niño trabajador son: tristeza, cansancio, mala alimentación y capacidad de aportar dinero a la casa. Como se ejemplifican en las siguientes citas: "preocupado, triste, por si vendió o no vendió" (madre, 29 años, SJL); "por la misma razón que trabaja se les ve cansaditos, ya no pueden estudiar, a veces se quedan dormidos" (madre, 33 ańos, SJL).

Asimismo, desde el punto de vista de las madres, las actividades que los(as) nińos(as) realizan se relacionan, por un lado, con responsabilidades en el hogar, como tender su cama, limpiar su cuarto y lavar lo que ensucian. De esta manera, desde muy temprana edad, muchos niños y niñas suelen cooperar en las tareas domésticas (OIT, 2003). Esto se ve reflejado en la siguiente cita: "En el hogar, yo considero que sea esa actividad sobretodo su orden, su cuarto, lo que es sus cosas (...) eso es lo principal que debe tener un niño" (madre, 52 años, VES).

Otras actividades propias de la infancia desde la perspectiva de los padres y las madres, se relacionan con el juego y la educación (OIT, 2007; Silva, 2010). En concordancia con esto, las madres entrevistadas consideran que los(as) nińos(as) realizan actividades recreativas como jugar y ver televisión: "Jugar, divertirse. Porque eso es lo que hace mi hijo, jugar, después se aburre, va a mirar tele" (madre, 27 ańos, VES). Así también, realizan actividades educativas, las cuales son consideradas importantes por los beneficios que se les atribuyen (Rausky, 2008). Para las madres, estos beneficios son: aprendizaje, adquisición de nuevos conocimientos; satisfacción familiar, alegría que sienten de que sus hijos(as) estudien; oportunidad de tener un mejor futuro, poder llegar a ser profesionales; y distancia de conductas de riesgo, como lo son los vicios. Esto se ve ejemplificado en los siguientes comentarios: 
"Porque al estudiar, ellos aprenden más, si no estudiaran ellos serían unos vagos, estarían en las cosas fumando, robando, en cambio al estudiar, ellos tienen métodos de conocimiento, según los años que vayan pasando, van logrando más conocimiento" (madre, 45 años, VES), "Uno se siente feliz viendo a su hijo estudiar, porque si no lo mandas a estudiar crece sin saber nada, a veces ni leer" (madre, 34 ańos, VES).

Así también, las creencias que los adultos tienen sobre la infancia, es decir, las características, necesidades y problemáticas que le son atribuidas, condicionan la manera en cómo los padres y madres se relacionan con los niños y niñas y las expectativas que se tienen sobre ellos (Alzate, 2003; Casas, 1998). Sobre esto, las madres entrevistadas evidencian tener tres expectativas compartidas sobre los(as) niños(as): que sean buenos padres/madres a futuro; que sean personas de bien, es decir que sean lo que ellas consideran como una buena persona; y que sean profesionales. Este último vinculado al beneficio que otorga la educación, de tener un mejor futuro. Lo que se ejemplifica en el siguiente comentario: "Nosotros de los niños esperamos que sean buenos muchachos, que tengan una buena profesión y que sean buenos padres" (madre, 36 años, VES).

Como se ha seńalado, conocer las creencias en torno al concepto de infancia es favorable para tener una mejor comprensión del significado que se le atribuye al trabajo infantil (Romero et al., 2012), el cual es definido como la participación de niños, niñas y adolescentes en actividades que contribuyen a la economía familiar, sin que sean necesariamente remuneradas (OIT, 2007; Silva, 2013). Con ello, es importante mencionar que gran parte de las actividades que se consideran trabajo infantil toman lugar en la calle, fuera del hogar. Estas pueden ser: vender productos como caramelos y flores; cuidar o lavar carros, lustrar zapatos, trabajar en puestos de mercados o tiendas, hacer espectáculos en la calle, etc. (Leyra, 2005; OIT, 2013; Sandoval, 2011). En concordancia con lo expuesto, las madres entrevistadas consideran vender golosinas, reciclar, limpiar carros, ser cobrador, cargar cosas, ayudar en restaurantes y mendigar como trabajo infantil. Como se observa en los siguientes comentarios: "Trabajo infantil, un niño vendiendo, subiéndose a los carros, los niños recicladores, si no niños que se 
paran en la esquina y piden colaboración" (madre, 36 años, VES); " $a$ veces la gente lo manda a vender cualquier cosa (...) yo veo a los niños con su carretilla" (madre, 36 años, SJL).

Por otro lado, otra forma que toma el trabajo infantil es el de labor doméstica, este refiere a cuando los niños, niñas y adolescentes realizan tareas propias del hogar como limpiar, lavar, planchar, preparar comida, cuidar hermanos menores (Calderón, 2004; OIT, 2007). Un ejemplo de labor dentro del hogar puede apreciarse en el siguiente comentario: "Que cuide a su hermano, porque dejar a uno con un hermano, ir a trabajar los padres, también es un trabajo, porque el hermano va a estar molestando y no va a tener tiempo de hacer nada" (madre, 27 años, VES).

Como se advierte, aunque existe un consenso, en las madres entrevistadas, acerca de considerar trabajo a las actividades productivas que realizan los(as) niños(as) fuera del hogar y que proporcionan un ingreso; en el caso de las actividades realizadas dentro del hogar, existe ambigüedad sobre lo que sí es o no trabajo infantil. Esto se debe a que, como se mencionó líneas arriba, algunas de las actividades realizadas por los(as) niños(as) dentro del hogar, son consideradas responsabilidades propias del infante: "Hacer sus deberes al levantarse de la cama, tender su cama, hacer su tarea, ordenar, atender a sus animales. Por ejemplo, mi hijo tiene deber de atender a sus cuyes, sus gatos, sus perros" (madre, 52 años, SJL). Esto coincide con lo expuesto por Silva (2010) y Román y Murillo (2013), quienes hacen referencia a que algunas de las actividades laborales realizadas por los niños y niñas, pueden ser consideradas ayuda o apoyo a la familia, ya que no existe una línea clara entre actividad laboral y apoyo al hogar.

Dentro de las causas asociadas al trabajo infantil, se reportaron, por un lado, causas vinculadas a los bajos ingresos económicos familiares. Y es que la razón que más se menciona como justificación del trabajo infantil es la necesidad económica (Acevedo, Quejada \& Yánez 2011; del Rio \& Cumsille, 2008; Silva, 2010). En este sentido, es dentro de la familia donde se estaría fomentando el trabajo en los(as) nińos(as), como parte de una estrategia que los incentiva a generar ingresos (Cerqueira $\&$ Neder, 2001), como se evidencia en el siguiente comentario: 
"Mayormente la gente le hace trabajar a sus hijos porque no tiene dinero" (madre, 33 años, SJL).

Otra razón reportada por las madres entrevistadas, se relaciona con la estructura familiar, pues mencionan que una de las causas por las que un niño(a) trabaja tiene que ver con provenir de un hogar monoparental o de que sus padres sean divorciados. Esto coincide con lo encontrado por Silva (2010) sobre la existencia de una expectativa de solidaridad, común en hogares monoparetales, donde la posibilidad de afrontar el gasto familiar es baja, por lo que necesitarían del apoyo de un ingreso extra por parte de los(as) hijos(as). Ejemplificado lo dicho: "Una razón puede ser que hay padres que son separados y el niño quiere ayudar a sustentar ganando un pequeño centavo, para ayudar a sus hermanitos, algo asi, vendiendo caramelo, para que haya una entrada para su hogar" (madre, 45 años, VES).

En casos más extremos, las entrevistadas mencionan que el trabajo infantil puede ser causado por presión familiar. En referencia a ello, Silva (2008) encontró que una de las razones por las que un nińo es castigado se relaciona con la actividad laboral que desempeña, es decir, si no trae suficiente dinero a la casa o si no realiza bien su trabajo. Una de las madres comenta:

Una vez yo pregunté: ¿y tu mamá?, “mi mamá está en la casa”, y ¿̇tu papá?, "también mi papá está en la casa”, y ¿te mandan a trabajar?, "sí, si yo no llevo la plata a la casa me botan, no me dan de comer, me hacen dormir en el suelo" (madre, 34 ańos, VES).

Por otro lado, otro de los motivos por los cuales los niños, niñas $\mathrm{y}$ adolescentes suelen trabajar, es para adquirir sus propios ingresos, es decir, para satisfacer necesidades personales. Es así que, un niño(a) estaría laborando para poder cubrir sus propios gastos que de otro modo no podrían ser cubiertos, ya que no están al alcance de la economía familiar (Cerqueira \& Neder, 2001; Silva, 2009). Esto concuerda con lo expresado en la siguiente viñeta: "Un niño trabajador será pues porque lo necesita (...) o su mamá o su papá no le da lo que necesita de lo que él quiere comprarse" (madre, 52 años, VES). 
Respecto a las consecuencias del trabajo infantil, las consecuencias positivas que mencionaron las entrevistadas, refieren principalmente al ingreso económico: "Su sustento diario puede ser un beneficio" (madre, 45 años, VES) y aprender a trabajar desde temprana edad "tiene un beneficio de que el niño aprenda a temprana a trabajar y a conocer su valor" (madre, 52 años, SJL).

Como consecuencias negativas, mencionan que existe un riesgo para el desarrollo del nińo, ya que el trabajo infantil afecta el tiempo dedicado a las actividades de recreación. En este sentido, lo que preocupa es que se privaría a los niños y niñas de su infancia al trabajar desde muy temprana edad (Calderón, 2004; OIT, 2003; Padrón \& Román, 2013), lo que se observa en la siguiente viñeta: "Está perdiendo una formación, su tiempo de niñez en que pueda jugar, pueda divertirse, asi como los demás niños, para mí no está bien que un niño trabaje" (madre, 27 años, VES). Esto coincide con lo hallado por Silva (2010), quien encontró que el trabajo infantil, es visto por los padres y madres como incompatible con las actividades propias de la infancia, como lo es el juego.

Asimismo, de acuerdo a las entrevistadas, existe un riesgo para el bienestar de los niños y niñas que laboran. En este sentido, el trabajo infantil puede ser nocivo para el desarrollo físico y mental, ya que puede llegar a perjudicar la salud, sobre todo cuando trabajan excesivas horas o cuando lo hacen en lugares peligrosos (Acevedo et al., 2011; Calderón, 2004; Padrón \& Román, 2013). Esto se aprecia en las siguientes vińetas: "En la calle corren riesgo, le puede atropellar el carro, hay personas malas que pueden agarrar a las niñas o niños, corren riesgo, lo pueden violar, muchas cosas pasan, lo matan, lo pueden tirar por el basurero" (madre, 34 años, VES); "les podría pasar cualquier cosa, están expuestos a muchos peligros, la gente le puede hacer daño, les pueden engañar, meterlos a los carros, es que los niños no miden las consecuencias" (madre, 33 ańos, SJL).

Otra consecuencia negativa es la adopción de conductas de riesgo, es decir, la implicación de los(as) niños(as) en actividades delictivas, o en actividades vinculadas con la drogadicción y el alcoholismo que 
dañan su salud y sus relaciones interpersonales. Sobre ello se presenta el siguiente comentario: "Asi ve a sus amiguitos que se van a trabajar, pero esos niñitos se han perdido bastante, ya empiezan con la droga, dejan el estudio, por su vicio de la drogadicción" (madre, 45 años, VES). Sobre la inserción en conductas de riesgo, podría decirse que se opone a la expectativa que tienen las madres sobre los(as) niños(as), respecto a que sus hijos(as) sean personas de bien. Asimismo, se ve opuesta al beneficio que da la educación, la cual implica una distancia de la misma, como se menciona líneas arriba.

Una de las consecuencias negativas que preocupan más a los padres y madres, es que el trabajo infantil puede interferir en la escolarización de los menores (Calderón, 2004; OIT, 2013; Padrón \& Román, 2013). En este sentido, las madres entrevistadas, refieren que el trabajo infantil puede llegar a afectar la educación de los(as) niños(as), respecto al tiempo que ellos le dedican a sus estudios:

"A un nińo lo mandas a la calle, aprende a muy temprana edad a tener dinero, y eso es el mal camino por el que se va, tienen dinero, ya no quieren estudiar, dejan la educación a un lado, y ya, "mamá sabe qué yo voy a trabajar" porque con ese dinero ya puede comprarse ropa, ya no depende de uno" (madre, 52 ańos, SJL).

Efectivamente, entre los investigadores se discute una posible incompatibilidad entre la escuela y el trabajo, ya que sostienen que cuando un nińo labora, puede abandonar el colegio o mostrar un bajo rendimiento escolar (Calderón, 2004; Rausky, 2008). Esto es algo comentado por las madres entrevistadas, ya que para ellas, el menor no tendría la capacidad de poder estudiar y trabajar al mismo tiempo, puesto que hacer ambas actividades distraería o cansaría a los(as) nińos(as): "un niño que trabaja no puede estudiar al mismo tiempo, porque no se concentra en sus estudios, porque ya está pensando en la hora que va a ir a trabajar no le alcanza el tiempo" (madre, 27 ańos, VES); "podría estudiar, pero no a su 100\%, porque estaría enfocado en otras cosas, en vender por ejemplo" (madre, 29 años, SJL). 
En contraste, otras investigaciones plantean que los valores sociales vinculados al trabajo pueden llevar a las personas a pensar que, a la larga, la labor infantil no tiene consecuencias perjudiciales respecto a las oportunidades que tenga el niño de progresar a futuro. Incluso de acuerdo a la OIT (2007) un porcentaje considerable de las personas entrevistadas opina que los niños y niñas que trabajan pueden llegar a tener mejores oportunidades de progresar que los niños y nińas que no trabajan. Asimismo, en un estudio hecho por Silva (2010), encontró que en el caso de la labor doméstica, los padres y madres consideran que esta tiene una función formativa para la vida a futuro del menor. Lo expuesto revela que existe una falta de consenso e incluso de conocimiento sobre las consecuencias a largo plazo que tiene el trabajo infantil. Es así que en este estudio las madres participantes no expresaron opiniones respecto a las consecuencias a largo plazo que podría tener el trabajo infantil.

En un estudio hecho en el Perú sobre las actitudes y vivencias de padres y madres de niños(as) que trabajan, se evidenció una tendencia hacia una valoración negativa y una actitud ambivalente hacia el trabajo infantil (Silva, 2009). Respecto a la percepción sobre la labor infantil, en zonas urbanas, se evidencia básicamente que esta es negativa (Silva, 2009; OIT, 2007). En este caso, sobre la evaluación en general que tienen las madres entrevistadas sobre el trabajo infantil, van desde ambivalente a negativo. Como se puede ejemplificar en los siguientes comentarios: "Yo creo que los niños no deberían trabajar tan pequeños, solo apoyar en sus casas con cosas que puedan hacer de acuerdo a su edad" (madre, 33 años, SJL); "La verdad yo lo veo bien mal que un niño trabaje, porque ellos no están para trabajar. Si uno trae al mundo a los hijos es para darle de comer, ellos no deberían trabajar" (madre, 33 años, SJL).

"Como balanceado, por una parte yo veo que sí ayuda, mi hija por ejemplo estudia, y los fines de semana, se iba a trabajar con mi mamá que tenía una bodega y ella le ayudaba y se ganaba su propina, por ese lado sí ayuda, pero sí también un poquito perjudica al estudio, porque le falta tiempo, o sea, no se concentra" (madre, 42 años, VES). 
Esto concuerda con lo hallado por Silva (2010) quien encontró que padres y madres residentes en Lima presentan una mayor valoración negativa hacia el trabajo infantil y una menor valoración positiva, ya que lo estarían asociando más a efectos negativos que positivos. Y respecto a una actitud ambivalente, esta se estaría presentando ligeramente con mayor frecuencia en Lima en comparación a otros departamentos (Silva, 2009).

Cabe mencionar, que el trabajo infantil puede ser visto como un fenómeno social, asociado a ciertos espacios geográficos caracterizados por pobreza, desempleo o falta de oportunidades (del Rio \& Cumsillle, 2008; Romero et al., 2012). Sobre el contexto en que viven, desde la perspectiva de las entrevistadas, mayormente en el caso de Villa El Salvador, este es considerado como un lugar inseguro tanto para las personas ajenas a la comunidad como para los(as) niños(as), razón por la cual verían las calles como peligrosas:

\begin{abstract}
"Un sitio tranquilo ha dejado de ser hace tiempo porque se ha vuelto peligroso. El otro día una chica que había venido, de las tiendas que vienen ofreciendo, la asaltaron allá, a las once de la mañana, la chica estaba llorando, le habían quitado todo los mismos mocosos de por acá" (madre, 36 años, VES).
\end{abstract}

Lo comentado en la cita es usual en contextos de pobreza, donde generalmente existe inequidad, baja cohesión social e inestabilidad familiar (Villarespe, Sánchez \& Espínola, 2012), las cuales pueden originar diversas formas de delincuencia como el robo, la estafa y el tráfico de drogas (Salinas, 2006). Lo que finalmente termina generando una sensación de inseguridad entre los habitantes de la comunidad (Sandoval, Palencia \& Martínez, 2009; Villarespe et al., 2012). Esta visión de las calles como poco seguras, puede influir en la percepción negativa que se tiene sobre el trabajo infantil, dado que la calle es considerada peligrosa, y un niño en este tipo de espacios se estaría arriesgando su bienestar y desarrollo (Sandoval, 2011). Por el contrario, en las entrevistas no se menciona que las actividades laborales dentro del hogar puedan ser dañinas. Esto puede deberse a que suelen ocurrir bajo la 
supervisión familiar; contrario a las actividades en la calle (Silva, 2009). Tomando en cuenta esto, las madres entrevistadas expresan el deseo de que sus hijos(as) no permanezcan dentro del contexto en el que viven y, por el contrario, construyan su futuro en otro lugar:

"Queremos que nuestros hijos sean profesionales, que salgan de acá, por ejemplo, yo le digo a mis hijos que yo no quiero que mis nietos crezcan en este arenal, yo quiero que mis nietos salgan, que vivan en otro lugar, por supuesto que todos los papás piensan eso" (madre, 36 años, VES).

Como se evidencia, existe un deseo y una expectativa por parte de las madres de que sus hijos(as) tengan un mejor futuro, y por características del contexto en el que viven actualmente, no consideran que este mejor futuro puedan lograrlo ahí. Cabe resaltar, como se mencionó anteriormente, este mejor futuro, de acuerdo a las madres, podría ser logrado a partir de sus estudios, mas no trabajando.

\section{Estructura axiológica de las participantes (Valores)}

Para comprender las creencias y actitudes hacia la infancia y el trabajo infantil, se ha tomado en cuenta como base los valores de los participantes, los cuales emergieron a partir de las entrevistas y fueron categorizados para su análisis de acuerdo al modelo de Gouveia et al. (2010). En este sentido los valores identificados como más importantes para las entrevistadas son: Trabajo y Progreso, los cuales corresponden, según el tipo de motivador, a valores materialistas, pues implican la orientación a metas específicas. Sobre estos valores, se puede decir que son comunes en contextos de escases económica, en donde las personas se orientan a garantizar condiciones necesarias para la supervivencia (Gouveia et al., 2010).

El Trabajo como valor, según el tipo de orientación es central, ya que no se focaliza exclusivamente en la sociedad o en sí mismo, pues de acuerdo a lo que mencionan las entrevistadas, las personas trabajan por ellas mismas y también por sus familias. Este valor es relevante para las madres debido a las causas y beneficios asociados a él, ya que una 
persona trabajadora puede obtener un ingreso económico que le permita satisfacer necesidades básicas: "Ser trabajador es importante, sin el trabajo, al menos acá en la ciudad no podría vivir, el que no trabaja, pues no come, no podría ni vestirse" (madre, 31 años, VES); cumplir metas específicas: "Seguir construyendo mi casa, ya están creciendo, cada quien tiene su cuarto, entonces seguir construyendo, seguir haciendo algo para ellos, para su futuro" (madre, 36 ańos, VES); y pagar la educación de sus hijos(as): "Para mis hijas que están estudiando, tengo que darles. Yo tengo que trabajar para que de repente mis hijas no sean como yo" (madre, 36 años, SJL). Asimismo, es considerado un medio para prevenir y combatir el estrés: "A veces estar todo el día en la casa también te estresa y si te estresa tanto no puedes (...) en cambio en mi trabajo yo me distraigo, veo otras personas, salgo del estrés" (madre, 36 años, VES).

La gran importancia que le dan las madres al Trabajo como valor, por un lado, podría explicar la importancia que le dan a la educación (Figueroa, 2004; Rausky, 2008), ya que una persona trabajadora puede financiar los estudios de sus hijos(as). Por otro lado, podría influenciar la justificación del trabajo infantil, al ver el ingreso económico como beneficio de la labor del menor (Acevedo et al., 2011; Calderón, 2004; Padrón \& Román, 2013).

El segundo valor de gran relevancia para las madres entrevistadas es el Progreso, el cual refiere a la mejora que se espera que busque una persona. De acuerdo al tipo de orientación es personal, pues se centra en atributos de realización y logros particulares de una persona (Gouveia et al., 2010). Ejemplificando lo mencionado: "salir adelante, progresar, ser mejor que nuestros padres y que nuestros hijos sean mejores que uno" (madre, 33 ańos, SJL). En el caso de un adulto, se espera que este progrese a partir de su trabajo, mientras que, en el caso de un niño, se espera que este progrese a partir de sus estudios, por lo que explicaría la importancia de la educación como medio para conseguir un futuro mejor (Figueroa, 2004; Rausky, 2008); así también explicaría la actitud negativa hacia el trabajo infantil, ya que este es considerado incompatible con los estudios (OIT, 2003; Padrón \& Román, 2013; Silva, 2009). En este sentido, el Progreso se relaciona con el Trabajo como 
valor, puesto que ambos tienen como fin la consecución de una meta específica, que sería la obtención de un mejor futuro.

Un valor central es la Responsabilidad, el cual según el tipo de motivador es humanitario, pues sirve como principio y criterio universal; y según el tipo de orientación es central, ya que no es exclusivamente focalizado en la sociedad o en sí mismo, puesto que la persona espera responsabilidad por parte de ella misma, al igual que de los miembros de su familia y de la comunidad (Gouveia et al., 2010). Para las madres, un adulto debe cumplir con sus obligaciones en relación al apoyo que brinda a sus hijos(as), y la preocupación que muestra hacia su comunidad en búsqueda de una mejora. Sobre esto último: "Una persona responsable, que se preocupa por su comunidad, va a hacer sus preguntas, sus reclamos a la municipalidad' (madre, 45 años, VES). Asimismo, las madres consideran la Responsabilidad como un valor importante también en los(as) niños(as) ya que sería necesario para realizar exitosamente actividades educativas (OIT, 2007; Silva, 2009). Sobre lo mencionado: "Es importante cuando es puntual, digamos está al día en su tarea (...) es responsable y logra tener buenas calificaciones" (madre, 45 ańos, VES). Y actividades relacionadas con sus responsabilidades dentro del hogar como ordenar su cuarto y lavar lo que ensucian (OIT, 2003). Como se puede advertir, la Responsabilidad se relaciona con valores como el Trabajo y la Filiación, en la medida que una persona actúa según sus obligaciones para con su familia y comunidad.

Otro valor según el tipo de motivador humanitario es: la Filiación, el cual expresa ideas universales en relación a la importancia de la familia, la solidaridad y la gratitud; y según el tipo orientador es social ya que, dentro del grupo familiar, en relación a los(as) hijos(as), se centran en regular y mantener las relaciones interpersonales; y dentro de la comunidad, entre sus miembros, se busca establecer una buena convivencia. Lo que significa que como subfunción entra en la categoría de Interactiva, en el que se expresa la necesidad de experiencia afectiva (Gouveia et al., 2010). Sobre la Filiación, las madres valoran el tiempo dedicado a sus hijos(as), tanto en actividades recreativas como educativas, es decir, pasando tiempo de recreación con ellos o ayudándolos en sus tareas: 
"Como mamá, los días que estoy en mi casa, me gusta dedicarme a ellos, hay veces digo no voy a salir, a veces mis amigas me dicen que vamos a tal sitio, y yo digo no, que no puedo, no tengo tiempo, porque me gusta estar en mi casa, me gusta estar con ellos, con mis hijos, porque son pequeños" (madre, 36 años, VES).

Lo anterior explicaría una actitud negativa hacia el trabajo infantil, ya que este implica menos tiempo compartido entre padres, madres e hijos(as).

\section{Conclusiones}

Advirtiendo la importancia de conocer y dar a conocer las creencias, actitudes y valores en torno a la infancia y trabajo infantil, y frente a la falta de información en el Perú sobre el cuidador principal de los infantes en contextos vulnerables y su manera de entender y evaluar dicha problemática, el presente estudio buscó identificar y analizar las creencias y actitudes entorno a la infancia y trabajo infantil y su relación con los valores individuales en madres dentro de un contexto de vulnerabilidad en Lima Metropolitana.

Sobre lo encontrado se concluye que, la infancia se ve relacionada con determinadas características y actividades vinculadas principalmente con la recreación y la educación. Sobre el trabajo infantil, las madres reconocen dos ámbitos en los que toma lugar: en la calle, donde todas las actividades son reconocidas como dañinas; y dentro del hogar, donde existe una ambigüedad respecto a que sería considerado trabajo infantil o responsabilidades de los(as) niños(as) en su hogar. Dentro de las causas atribuidas al trabajo infantil, se encuentra, por un lado, el incentivo familiar como estrategia económica, y por otro lado, la motivación propia del niño para cubrir sus necesidades. Sobre las consecuencias, todos se perciben como negativas en el corto plazo, por lo que finalmente la madre tendría una evaluación principalmente negativa al trabajo infantil y ambivalente en algunos casos. Reconocer este conjunto de ideas, creencias y actitudes que se tiene sobre el trabajo infantil es de suma importancia, ya que influencia en cómo la labor infantil es conceptualizada y cómo se actúa frente a esta. 
Sobre el contexto de vulnerabilidad en el que viven, podría decirse que este puede influenciar sus actitudes sobre la infancia y el trabajo infantil. La percepción de las calles como poco seguras estaría influyendo en una percepción negativa sobre el trabajo infantil, ya que en tanto sean consideradas peligrosas, un niño en este tipo de espacios se estaría arriesgando su bienestar y desarrollo. Asimismo, las problemáticas existentes en la comunidad, como es el caso de la delincuencia, estarían influenciando en las creencias de las madres sobre lo que es mejor para el desarrollo de sus hijos(as), ya que consideran que para que sus niños(as) progresen, no solo sería necesario que estudien, sino que salgan de la comunidad.

Por otro lado, uno de los hallazgos principales de estudio gira en torno a que los valores reportados por las madres consideran elementos a la base de lo que evidencia preliminar refiere, con respecto a las creencias y actitudes sobre el trabajo infantil, ya que estructurarían el modo en que dicha dinámica sería evaluada de forma ambivalente. Con ello, los valores identificados a partir del análisis son: el Trabajo, el Progreso, la Responsabilidad y la Filiación.

Sobre el Trabajo, este es considerado medular, ya que una persona trabajadora provee principalmente un ingreso económico que puede satisfacer necesidades básicas, ayudar a alcanzar metas específicas, y pagar la educación de los(as) hijos(as). Entonces la importancia que se le da al trabajo como valor, puede explicar la percepción de las madres de que el único beneficio de la labor infantil, sea el ingreso económico.

Sobre el Progreso, este explicaría la importancia que le otorgan las madres al estudio, ya que este se vería como medio por el cual podrían tener un mejor futuro, lo que se relaciona con las expectativas que las madres tienen sobre sus hijos(as) a futuro, de que lleguen a ser profesionales. Asimismo explicaría la percepción negativa del trabajo infantil, pues este afecta la escolaridad de los nińos(as).

La Responsabilidad, sería un valor central que se relaciona con valores como el Trabajo y la Filiación, ya que existe una expectativa de que las personas sean conscientes de sus deberes y actúen en función de ellas. En el caso de los adultos, se espera que sean ellos los que trabajen 
y apoyen a sus hijos(as), con sus estudios y dedicándoles tiempo; mientras que en el caso de los(as) niños(as) se espera que no trabajen, sino que cumplan con sus deberes, respecto a sus estudios y a sus responsabilidades en el hogar.

Respecto a la Filiación, es un valor en las madres que explicaría la actitud negativa hacia el trabajo infantil, ya que este supone que los(as) nińos(as) inviertan menos tiempo con sus madres, en tanto ellas ven como prioridad el tiempo compartido con sus hijos(as), tanto en sus actividades educativas como recreativas.

Como se advierte, los primeros valores están focalizados a la consecución de recursos que, finalmente, redundan no solo en el bienestar individual, sino también en el del grupo más cercano de parentesco, como lo son los(as) hijos(as). En ese sentido, se podría decir que los valores abren un marco sobre el cual se puede analizar la manera en que algunos grupos poblacionales desarrollan determinadas actitudes hacia problemáticas sociales como es el caso del trabajo infantil.

\section{Alcances del estudio, limitaciones y recomendaciones}

La presente investigación tuvo como principal objetivo identificar y analizar las creencias, actitudes entorno a la infancia y el trabajo infantil teniendo como base los valores individuales en las madres en un contexto de vulnerabilidad. Así dar algunas contribuciones a nivel teórico y a nivel práctico, teniendo en cuenta que un adecuado entendimiento del trabajo infantil es elemental para suprimir prácticas que son o pueden llegar a ser dañinas para el infante (Padrón \& Román, 2013). En este sentido, la información obtenida podría tomarse en cuenta en posibles programas e intervenciones vinculados al trabajo infantil, donde sería de utilidad conocer las creencias, actitudes y sobre todo, los valores de los cuidadores principales para tener un mayor entendimiento de dicha problemática y así incluirlos a como parte de la intervención, ya que la decisión de enviar o no a los(as) niños(as) a trabajar, lo toman generalmente los padres (Cerqueira \& Neder, 2001; Emerson \& Portela, 2003; Figueroa, 2004). 
Asimismo, el presente estudio podría ser de utilidad para desarrollar una investigación cuantitativa, tomando en consideración una muestra representativa a partir de la cual se puedan generalizar los resultados encontrados. En este sentido, una ampliación del estudio también podría servir como herramienta para iniciativas ya sea desde el Estado o alguna institución privada que vea este tipo de problemática, velando por la protección de los derechos de los niños y nińas.

En el caso del Estado, podría ser de utilidad dentro del Enfoque de Hogar de la Estrategia Nacional para la Prevención y Erradicación del Trabajo Infantil (ENPETI) planteado desde el 2012 hasta el 2021. El cual busca intervenir dentro de los hogares a través de diversas estrategias, como brindar información y generar mayor conocimiento en los padres en temas que afectan su toma de decisiones, en especial aquellas que involucran al niño y a sus actividades (MTPE, 2012). Por su parte, en el caso de organizaciones privadas, podría ser de utilidad dentro del marco del Proyecto Semilla, dirigido por Fundación Desarrollo y Autogestión, Desco y World Learning. Este proyecto tiene como fin la erradicación de las peores formas del trabajo infantil en zonas rurales, a través de intervenciones a nivel familiar, social y/o educativo, mediante la ejecución de talleres dirigidos a niños(as), padres y madres (Semilla, s/f).

En relación a las limitaciones de la presente investigación, la principal hace referencia a la falta de información por parte de una población masculina que hubiera permitido conocer sobre las creencias y actitudes de los padres, y realizar contrastes según el género, siendo ellos quienes son en la mayoría de los casos el jefe de hogar (INEI, 2013). Por lo que se sugiere incluir a los padres de familia en futuras investigaciones. Asimismo, hubiera sido útil contar con padres o madres con un menor nivel educativo para verificar si se cumple o no lo hallado por previas investigaciones en donde se plantea que a mayor nivel educativo, menor es la probabilidad de que se espere que el hijo contribuya en casa. Es así que se recomienda, previo al levantamiento de información, revisar los resultados de las encuestas realizadas por el INEI, con el fin de identificar aquellos contextos donde prima un menor nivel educativo en los padres y/o madres. 


\section{Referencias}

Acevedo, K., Quejada R. \& Yánez, M. (2011). Determinantes y consecuencias del trabajo infantil: un análisis de la literatura. Revista Facultad de Ciencias Económicas, 19(1), 113-124. https://doi. org/10.18359/rfce.2263

Alegre, M. J. \& Bielich, C. (2015). Vulnerabilidad urbana: evaluando los nuevos factores de riesgo en Lima Metropolitana. Lima: Oxfam. Alzate, M. (2003). La infancia: concepciones y perspectivas. Pereira: Papiro.

Arrizaga, C. (2010). Derechos del niño en contexto de vulnerabilidad social: percepciones de padres y profesores (Tesis de licenciatura). Escuela de Trabajo Social, Universidad Académica de Humanismo Cristiano, Santiago, Chile.

Bohner, G. \& Dickel, N. (2011). Attitudes and Attitude Change. Annual Review Psychology, 62, 391-417. https://doi.org/10.1146/ annurev.psych.121208.131609

Calderón, A. (2004). Trabajo infantil y adolescente en el Agro: ¿Necesidad o identidad? Revista de Ciencias Sociales, 106(4), 165-179.

Casas, F. (1998). Infancia: perspectivas psicosociales. Barcelona: Paidós.

Cerqueira, G. \& Neder, G. (2001). Social and historical approaches regarding street children in Rio de Janeiro (Brazil) in the context of the transition to democracy. Childhood, 8, 11-29. https://doi. org/10.1177/0907568201008001002

Del Rio, M. \& Cumsille, P. (2008). ¿Necesidad económica o preferencias culturales? La justificación parental del trabajo infantil en Chile. PSYKHE, 17(2), 41-52. https://doi.org/10.4067/ S0718-22282008000200004

Encuesta Lima cómo vamos (2015). Quinto informe de la percepción de la calidad de vida. Recuperado de http://www.limacomovamos.org/

Figueroa, B. (2004). No somos invisibles: resultados de consulta a trabajadores infantiles domésticos (TID) y ex trabajadores infantiles domésticos (EXTID) en Perú (ciudades de Lima, Cajamarca y Pucallpa). Lima, Perú: Asociación Grupo de Trabajo Redes. 
Garzón, A. (2012). Incorporación y adaptación del sistema de creencias postmodernas. Psicothema, 24(3), 442-448.

Gouveia, V. V. (2003). A natureza motivacional dos valores humanos: Evidências acerca de uma nova tipologia. [La naturaleza motivacional de los valores humanos: evidencias acerca de una nueva tipología]. Estudos de Psicologia (Natal), 8, 431-443. https://doi. org/10.1590/S1413-294X2003000300010

Gouveia, V. V., Fischer, R. \& Milfont, T. L. (2009). A functional theory of terminal values: Testing content and structure hypotheses. Manuscript submitted for publication.

Gouveia, V., Santos, W., Milfont, T., Fischer, R., Clemente, M. \& Espinosa, P. (2010). Teoría funcionalista de los valores humanos en España: comprobación de las hipótesis de contenido y estructura. Revista Interamericana de Psicología, 44(2), 213-224.

Graneheim, U. H. \& Lundman, B. (2004). Qualitative content analysis in nursing research: concepts, procedures and measure to achieve trustworthiness. Nurse Education Today, 24, 105-112. https://doi.org/10.1016/j.nedt.2003.10.001

Hernández Sampieri, R., Fernández Collado, C. \& Baptista Lucio, P. (2014). Metodología de la investigación: Roberto Hernández Sampieri, Carlos Fernández Collado y Pilar Baptista Lucio (6a. ed.). México D.F.: McGraw-Hill.

Holland, R.W., Meertens, R.M. \& Van Vugt, M. (2002). Dissonance on the road: selfesteem as a moderator of internal and external self-justification strategies. Personal Social Psychology, 28, 1713-1724. https://doi.org/10.1177/014616702237652

Instituto Nacional de Estadística e Informática (Perú) (2015). Mapa de pobreza provincial y distrital 2013. Lima: Instituto Nacional de Estadística e Informática (Perú). Recuperado de http://repositorio.minedu.gob.pe/handle/123456789/3638

Kilby, R. (1993). The study of human values. Lanham, MD: University Press of America.

Leyra, B. (2005). El trabajo infantil en México: reflexiones de una antropóloga. Revista de Antropología Iberoamericana, 40, 1-5. 
Liebel, M. (2003). Infancia y Trabajo. Lima: Ifejant.

Lightfoot, C. \& Valsiner, J. (1992). Parental belief systems under the influence: Social guidance of the construction of personal cultures. En I. E. Sigel, A. V. McGillicuddy-DeLisi \& J. J. Goodnow (Eds.), Parental belief systems: The psychological consequences for children (pp. 393-414). Hillsdale, NJ: Lawrence Erlbaum.

Ministerio de Trabajo y Promoción de Empleo (MTPE) (2012). Estrategia nacional para la prevención y erradicación del trabajo infantil 2012-2021. Lima: Mintra.

Organización Internacional del Trabajo (2003). Combatiendo las peores formas del trabajo infantil: manual para inspectores. San José: OIT. Organización Internacional del Trabajo (2007). Trabajo infantil: estudio de opinión pública en el Perú. Lima: OIT.

Organización Internacional del Trabajo (2013). Medir los progresos en la lucha contra el trabajo infantil - Estimaciones y tendencias mundiales entre 2000 y 2012. Ginebra: OIT.

Oliveira, A. \& Uchoa, A. (2010). Iguales, pero diferentes: creencias sociales en la canalización cultural del desarrollo de gemelos. Revista de Psicología, 28(2), 341-380.

Padrón, M. \& Román, P. (2013). Particularidades y complejidades del trabajo infantil: aspectos conceptuales y aproximación empírica a un fenómeno oculto con dimensiones diversas. Revista Facultad Ciencias Económicas, 21(1), 25-42. https://doi.org/10.18359/ rfce. 665

Rausky, M. (2008). Acerca de la relación trabajo infantil y asistencia escolar: Apuntes para su comprensión. Questión, 1(17).

Román, M. \& Murillo, F. J. (2013). Trabajo infantil entre los estudiantes de educación primaria en América Latina. Características y factores asociados. Revista Electrónica de Investigación Educativa, 15(2), 1-20.

Romero, V., Amar, J. J., Palacio, J., Madariaga, C., Sierra, E. \& Quintero, S. M. (2012). Factores familiares y sociales de alto riesgo asociados al trabajo infantil en ciudades de la Costa Caribe colombiana. Universitas Psychologica, 11(2), 481-496. https:// doi.org/10.11144/Javeriana.upsy11-2.ffsa 
Ruiz, N. (2012). La definición y medición de la vulnerabilidad social. Un enfoque normativo. Investigaciones Geográficas, Boletín del Instituto de Geografia, UNAM, 77, 63-74. https://doi. org/10.14350/rig.31016

Salinas, P. (2006). Pobreza y salud. Un problema global, sus causas, consecuencias y soluciones. Revista de Facultad de Medicina, Universidad de Los Andes, 15(1), 17-21.

Sandoval, L., Palencia, A. \& Martínez, D. (2009). Pobreza y delincuencia departamental en Colombia 2003-2007. Revista Facultad de Ciencias Económicas, 17(1), 95-108.

Sandoval, C. (2011). Erradicación del trabajo infantil en el perú: lineas estratégicas de comunicación. Extraído de http://www.facilitandocomercio.com/informes/laboral/laboral2

Schwartz, S. (2007). Basic human values: Theory, methods and application. Revista di Psicologia del Lavoro e dell'Organizzazione, 13(2), 261-283.

Silva, G. (2010). Voces sobre el trabajo infantil: actitudes y vivencias de padres, madres y maestros de niños que trabajan. Lima: Instituto de Estudios Peruanos.

Silva, M. (2013). El abordaje del trabajo infantil desde la indagación de la familia: un aporte a las políticas públicas. Revista de Ciencias Sociales, 19(3), 536-546.

Thurmond, V. (2001). The point of triangulation. Journal of nursing scholarship, 33, 253-258. https://doi.org/10.1111/j.1547-5069. 2001.00253.x

Villarespe, V., Sanchez, A. \& Espínola, M. (2012). Pobreza e inseguridad el viejo debate entre desarrollo y represión: un enfoque empirico. México: UNAM

Ward, C., Bochner, S. \& Furnham, A. (2001). The Psychology of Culture Shock. Routledge. Extraído de http://discovery.ucl.ac.uk/22886/

Recibido: 7 de julio. 2018 Revisado: 20 de febrero, 2019

Aceptado: 5 de agosto, 2019 